DOI: https://doi.org/10.46296/gt.v2i3.0006

\title{
CALIDAD DE VIDA Y SATISFACCIÓN LABORAL DE LOS FUNCIONARIOS DEL ÁREA DE FARMACIA DEL HOSPITAL TEODORO MALDONADO CARBO, ECUADOR
}

\section{QUALITY OF LIFE AND JOB SATISFACTION OF WORKERS IN THE PHARMACY AREA OF THE TEODORO MALDONADO CARBO HOSPITAL, ECUADOR}

\author{
Mendoza-Mestanza Geanella Vanessa ${ }^{1 *}$ \\ ${ }^{1}$ Universidad Católica de Santiago de Guayaquil, UCSG. Guayaquil, Ecuador.
}

*Correo: gea-mendoza-923@gmail.com

\begin{abstract}
Resumen
La satisfacción laboral en las organizaciones es un tema de importancia ya que repercute no sólo en los resultados de la empresa, sino que también en diferentes aspectos de los colaboradores, como lo es la calidad de vida. El objetivo general de este trabajo es determinar la incidencia de la satisfacción laboral y la calidad de vida de los funcionarios del área de Farmacia del Hospital Teodoro Maldonado Carbo. La metodología utilizada fue en base a un diseño cuantitativo, con método deductivo, de tipo descriptiva, correlacional y de corte transversal. Se realizó un censo a la totalidad de la población que fue de 75 empleados, las dimensiones de calidad de vida analizadas fueron salud, felicidad, satisfacción con la vida, satisfacción económica, satisfacción con la vida familiar con dos escalas y satisfacción con el bienestar subjetivo, en tanto que la satisfacción laboral fue medida en base a dos constructos. Se comprobó que todas las dimensiones de calidad de vida están positivamente correlacionadas con las dos escalas de satisfacción laboral.
\end{abstract}

Palabras clave: satisfacción, calidad, trabajadores, hospital.

\begin{abstract}
Job satisfaction in organizations is an issue of importance because it affects not only the company's results, but also in different aspects of employees, such as quality of life. The general objective of this work is to determine the incidence of job satisfaction and quality of life of the officials of the Pharmacy area of the Teodoro Maldonado Carbo Hospital. The methodology used was based on a quantitative design, with a deductive method, descriptive, correlational and crosssectional. A census was made to the entire population that was 75 employees, the dimensions of quality of life analyzed were health, happiness, satisfaction with life, economic satisfaction, satisfaction with family life with two scales and satisfaction with subjective well-being, whereas job satisfaction was measured based on two constructs. It was found that all dimensions of quality of life are positively correlated with the two scales of job satisfaction.
\end{abstract}

Keywords: satisfaction, quality, workers, hospital.

Información del manuscrito:

Fecha de recepción: 09 de noviembre de 2018

Fecha de aceptación: 21 de diciembre de 2018

Fecha de publicación: 10 de enero de 2019 


\section{Introducción}

La calidad de vida se refiere a la forma de vivir de un individuo, la misma hace alusión a los estados de ánimo de una persona de acuerdo a diferentes aspectos de la vida. La calidad de vida está relacionada con varias posturas que retumban en lo cotidiano, con esto se quiere decir que el estilo de vida de una persona está afectado por situaciones políticas, familiares y en su gran mayoría está definido por la salud. En general, la calidad de vida tiene que ver con lo material, lo físico, lo social, la superación, el enfoque, la actividad, los procesos y las emociones; la calidad de vida tiene un comportamiento cambiante, su desenvolvimiento radica en el bienestar 0 en el malestar de cualquier tipo de aspecto humano (Ávila, 2013).

La calidad de vida de una persona es un factor importante para los profesionales de la salud, puesto que a través de una oportuna evaluación logran tomar las decisiones correctas y más adecuadas para el propio beneficio de los pacientes. La evaluación de la calidad de vida de un usuario ayuda a que se le destine un tratamiento para superar todo problema que lo esté afectando tanto en la salud como en lo emocional y psicológico (Ávila, 2013).

La satisfacción laboral es el estado emocional que refleja un colaborador o profesional, la misma nace en el transcurso en que se está realizando algún tipo de trabajo en particular. La satisfacción en el puesto de trabajo comprende dentro de su terminología tres aspectos principales dentro de un ámbito funcional-técnico y empresarial, estos son: (a) el aspecto conductual; (b) el cognitivo; (c) el aspecto afectivo. El primero, se desarrolla al momento en que el individuo está desempeñándose en su actividad laboral, el segundo aparece cuando la persona refleja una percepción en base a una determinada actividad y el último está constituido por el sentimiento que tiene el colaborador hacia la actividad o trabajo que realiza (Lozada, 2015).

Es importante que exista satisfacción en los trabajadores; ya que de esto depende mucho su desempeño dentro del puesto que ocupa e inclusive su motivación. Cuando un trabajador encuentra la satisfacción en su trabajo tiende a tener un mejor desenvolvimiento y es más propenso 
a que ayude activamente a la empresa a que alcance sus objetivos dentro del mercado. La satisfacción en muchos casos no tiene que ver con lo monetario, sino que su desarrollo se expande dentro de un lugar de trabajo por los estímulos, el apoyo, la comprensión y la oportunidad de superarse que recibe el empleado por parte de una persona de rango superior (jefe) (Lozada, 2015).

El Hospital Teodoro Maldonado Carbo es un instituto de salud pública ubicado en la ciudad de Guayaquil, en la actualidad está experimentando problemas en sus procesos internos, específicamente en el área de farmacias. En general dentro de esta área se refleja desmotivación del personal por el cambio de jefes de forma inmediata, además existe una desvinculación de una farmacia con otra (farmacia de dosis unitaria con la de consulta), esto permite inferir que existen diferencias. Por consiguiente, el objetivo de la presente investigación es determinar la incidencia de la satisfacción laboral y la calidad de vida de los funcionarios del área de Farmacia del Hospital Teodoro Maldonado.

\section{Metodología}

El presente marco metodológico ha sido desarrollado tomando en cuenta como primera directriz a los tipos de investigación, en donde se explica que se utiliza el alcance descriptivo y el correlacional, posteriormente se toma en cuenta el método deductivo, como población de estudio se tomó en cuenta a todos los colaboradores del área de farmacia del hospital. La técnica de recolección de datos que se utilizó es la encuesta.

\subsection{Tipo de investigación}

Una investigación de mercado dentro de su metodología consta de un conjunto limitado de tipos de investigación, estas se definen en: descriptivas,

exploratorias, correlacionales y explicativas. La exploratorias son desarrolladas cuando el tema del proyecto no ha sido estudiado anteriormente, las correlacionales pretenden mostrar la incidencia de una variable de estudio con otra y a su vez permite probar la hipótesis de la investigación, las explicativas abordan diferentes conocimientos a través de la explicación de los datos obtenidos y la descriptiva tiene el objetivo de responder a las preguntas de investigación, partiendo de la 
descripción y especificación de las cualidades de un grupo de personas que forman parte del proyecto de investigación (Universidad de las Américas Puebla, 2018).

Para desarrollar el presente estudio se hizo uso del tipo de investigación descriptiva por motivo de que se estableció una caracterización de la situación actual del Hospital Teodoro Maldonado Carbo, con respecto a su área de farmacia que consta de la farmacia de dosis unitaria, farmacia de consulta externa, farmacia de quirófano, farmacia de Emergencia y farmacia de hospitalización. El segundo tipo investigativo que se usó fue el correlacional, ya que correlacionaron las variables para evaluar la incidencia entre las mismas, esto permitió probar la hipótesis establecida.

\subsection{Enfoque}

El desarrollo de una investigación está guiado por la implicación de dos enfoques muy particulares que establecen formas diferentes para llegar a establecer resultados importantes para conseguir resolver un problema de investigación. Los enfoques que intervienen son el cuantitativo y el cualitativo, la metodología de trabajo de estos enfoques son diferentes, sin embargo, ambos presentan cinco etapas homogéneas que se encuentran vinculadas entre sí: los dos enfoques se dedican a evaluar los diferentes fenómenos, ambos crean ideas 0 escenarios con respecto a lo que han visualizado y evaluado, mediante su evaluación prueban y muestran el nivel de fundamento de las ideas, analizan las ideas y sugieren nuevas observaciones y estudios para generar nuevas suposiciones y corregir las otras (Vega et al., 2014).

El enfoque cuantitativo fue el que se utilizó en el desarrollo de la investigación, la funcionalidad del mismo permitió obtener la información necesaria para incurrir en un estudio analítico de datos que permitió responder las preguntas de investigación y determinar el cumplimiento o incumplimiento de la hipótesis. La estructura que maneja este enfoque proviene de lo numérico y de lo estadístico, aspectos certeros para analizar el comportamiento de las personas (Vega et al., 2014).

\subsection{Método}

Una investigación está conformada por dos métodos, el inductivo y el 
deductivo, el primero de ellos se produce cuando se empieza de lo particular y se culmina en aspectos universales, mientras que el método deductivo se realiza cuando se desarrolla un proceso de estudio desde lo universal y se recae en lo particular (Instituto de Hematología e Inmunología, 2018). La investigación fue desarrollada utilizando el método deductivo, puesto que el estudio empezó de lo general como fue el análisis del marco teórico, luego se prosiguió a comprobar la hipótesis en base a los datos recopilados y finalmente se construyeron conclusiones particulares que ayudaron a resolver el problema investigativo. La investigación tuvo un corte transversal.

\subsection{Población}

Las personas consideradas para que formen parte del estudio fueron los auxiliares, químicos, un jefe y asistentes que se desempeñaban en el área de farmacia del Hospital Teodoro Maldonado Carbo de Guayaquil. El número total entre los auxiliares y químicos farmacéuticos es de 72 , además de un jefe de farmacia y dos asistentes administrativos, por lo tanto, se obtuvo un total de 75 colaboradores, cantidad de trabajadores que fue considerada para ser evaluada. Debido a la facilidad de acceso a la recopilación de los resultados se procedió a realizar un censo con la totalidad de los trabajadores.

\subsection{Técnica de recopilación de la información}

El cuestionario de la encuesta es un instrumento de investigación que pretendió recoger la información necesaria para dar respuesta a las preguntas de investigación (Instituto de Hematología e Inmunología, 2018).

El cuestionario que se utilizó fue la "Encuesta de calidad de vida en relación a la satisfacción laboral y estilo de vida", este cuestionario está conformado por 17 preguntas formuladas con el objetivo de determinar la relación existente entre la calidad de vida y la satisfacción laboral. El cuestionario fue elaborado a través de diferentes escalas, estas fueron: (a) el índice de calidad de vida relativo a la salud de Hennessy, Moriarty, Zack, Scherr y Brackbill (1994); (b) la escala de felicidad de Lyubomirsky y Lepper (1999) que fue traducida por Vera y Celis (2011); (c) escala de satisfacción con la vida de Diener et al. (1985) traducida por la 
Universitat de Valéncia (2018); (d) escala de satisfacción con la vida familiar de Zabriskie y Ward (2013); (e) escala de bienestar económico subjetivo de Giarrizzo (2010); (f) escala de satisfacción con la vida del Journal of Personality Assessment de Diener et al. (1985); (g) escala de satisfacción laboral de Warr (1999) traducida por Pérez y Fidalgo (1999); (h) escala de satisfacción laboral de Spector (1997).

A continuación, se explican las escalas más importantes tomadas en cuenta para medir la satisfacción laboral y calidad de vida de los colaboradores:

La escala del índice de calidad de vida relativo a la salud de Hennessy, Moriarty, Zack, Scherr y Brackbill (1994) se conforma por un reactivo acerca de cómo se considera la persona con respecto a su salud en general, el segundo reactivo se refiere a la salud física, el tercero a la salud mental y el cuarto y último reactivo se orienta a las actividades que no pudo realizar la persona, la respuesta del primer reactivo va desde muy mala (1) a excelente (5), en los demás reactivos como respuesta la persona tiene que colocar el número de días.
La escala de la felicidad muestra cuatro reactivos que tienen la finalidad de medir el estado de ánimo de la persona en base al valor de la felicidad, la dimensión se estructura en base a la escala de Likert que va desde una persona no muy feliz (1) a una persona muy feliz (7), el primer reactivo se refiere a "cómo se considera la persona", el segundo reactivo presenta una respuesta que parte desde menos feliz (1) a más feliz (7), el reactivo se refiera a "comparado en la mayoría de sus pares, usted se considera"; el tercer reactivo tiene que ver con una comparación del encuestado, se menciona que "si la persona disfruta de la vida sin importar de lo que pase" las respuestas van desde para nada (1) hasta totalmente (7), las respuestas del último reactivo son las mismas que el reactivo anterior, en este caso el reactivo se refiere a la no felicidad de la persona.

Las respuestas de la escala de satisfacción con la vida de Diener (1985) se componen por "en desacuerdo" y "de acuerdo", en esta escala existen cinco reactivos, el primero de ellos se refiere a que si la vida de la persona es la ideal, el segundo tiene que ver con que si las 
condiciones de vida son excelentes, el tercero con que si la persona se encuentra satisfecha con su vida, el siguiente se orienta a saber si la persona hasta el momento ha conseguido cosas importantes que ha deseado alguna vez y el último reactivo se refiere a que si la persona podría vivir de nuevo cambiaría algo.

En la escala de satisfacción con la vida familiar de Zabriskie y Ward (2013), las respuestas van desde totalmente en desacuerdo hasta totalmente de acuerdo, el primer reactivo se refiere a que si la vida familiar es la adecuada, el siguiente habla acerca de que si las condiciones de vida familiares son excelentes, el tercero hace referencia a que si la persona se encuentra satisfecha con su vida familiar, el cuarto indica que si hasta el momento la persona ha conseguido adquirir las cosas importantes que ha querido en su vida familiar y el último se refiere a que si la persona cambiaría algo de su vida familiar si pudiera vivir de nuevo.

La escala de satisfacción laboral de Warr (1999) traducida por Pérez y Fidalgo (1999) tiene que ver con todo lo referente al trabajo en el cual se desempeña la persona, por medio de esta escala se busca medir la satisfacción e insatisfacción de la persona con respecto al salario que percibe, su estabilidad, las condiciones físicas del empleo y demás aspectos.

La escala de satisfacción laboral de Spector (1997) tuvo el objetivo de medir el nivel de desacuerdo y de acuerdo de la persona con respecto a las oportunidades de superación profesional que se le brindan dentro del trabajo, los beneficios que recibe, acerca de la participación del jefe o supervisor, la comunicación, el reconocimiento, acerca del trato que existe entre el jefe, entre otros aspectos referentes.

\section{Resultados y discusión}

El análisis de resultados se procedió a dividir según la percepción de calidad de vida en la muestra estudiada.

\subsection{Calidad de vida desde la salud}

La calidad de vida según la salud fue determinada en base al primer ítem de la sección 1, en donde se señala que los encuestados consideraron su salud como excelente en un $14,67 \%$, muy buena en un $18,67 \%$, buena en un $36,00 \%$, regular en un 
$20 \%$ y muy mala en un $10,67 \%$. Los resultados reflejan que no existen mayores problemas en cuanto a la calidad de vida según la salud de las personas. El promedio en la escala de Likert de cinco valores fue de 4,09 , siendo mayor a la mediana de tres, por lo que se considera que la mayoría de colaboradores se encuentra bien de salud.

En las preguntas dos, tres y cuatro de la sección I se solicitó la cantidad de días que la persona tuvo problemas físicos y emocionales; además se preguntó cuántos días la persona quedó sin capacidad de realizar actividades comunes como la recreación. Se evidenció que un $24 \%$ de las personas tuvo un día con el problema, el $12 \%$ con dos días, mientras que el $64 \%$ no tuvo problemas; en cuanto a los problemas emocionales el $12 \%$ tuvo afectación un día en el mes, el 5,33\% dos días en el mes, mientras que el $82,67 \%$ no tuvo problemas; en cuanto a la incapacidad de realizar actividades comunes, el 18,67\% tuvo un día sin poderlas realizarlas, el $9,33 \%$ dos días, mientras que el $72 \%$ no tuvo problemas. La tabla 1 refleja los problemas que se han detectado en los trabajadores y el número de días respectivo.

Tabla 1.

Días con problemas físicos, emocionales e incapacidad

\begin{tabular}{cccc}
\hline Tipo de problema & 1 día & 2 días & 0 días \\
\hline Físico & $24.00 \%$ & $12.00 \%$ & $64.00 \%$ \\
Emocional & $12.00 \%$ & $5.33 \%$ & $82.67 \%$ \\
Incapacidad de & $18.67 \%$ & $9.33 \%$ & $72.00 \%$ \\
realizar acciones & & &
\end{tabular}

Nota: Datos tomados del procesamiento de datos en SPSS 22.

\subsection{Calidad de vida desde la felicidad}

En cuanto a la pregunta para determinar si la persona se considera feliz, con la escala de Likert de siete niveles, siendo el séptimo el ser muy feliz, se obtuvo que el $42,67 \%$ se ubicó en el nivel cinco; el tamaño de las barras señala que las personas estuvieron mayormente ubicadas del lado de mayor felicidad, el promedio general de esta pregunta fue 4,57 , siendo superior a la mediana. 
La figura 1 muestra los resultados

completos.

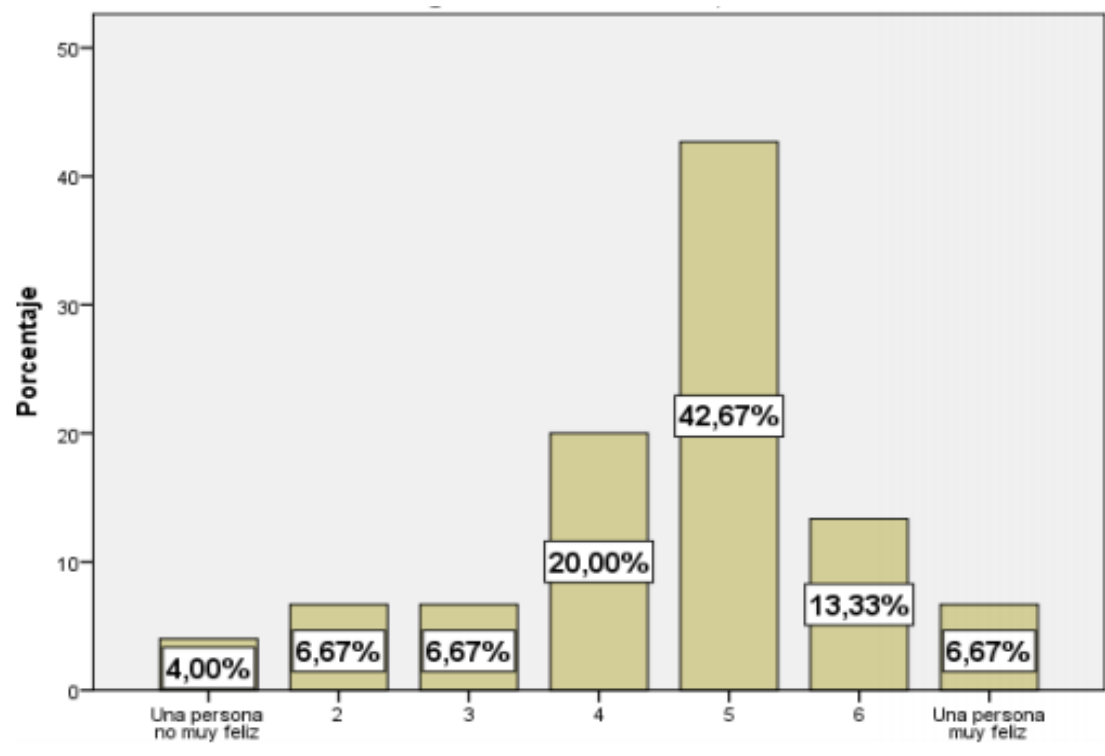

Figura 1. En general se considera

En lo que respecta a la felicidad analizada desde un punto comparativo con las demás personas que lo rodean, se identificó que el $54,67 \%$ se ubicó en la valoración de cinco, sobre las siete valoraciones presentadas, un 9,33\% se consideró más feliz, mientras que un $4 \%$ señaló ser menos feliz; el promedio de esta pregunta fue de 4,80 siendo mayor a la mediana, es decir hay mayor felicidad entre los colaboradores.

En la pregunta que evalúa la calidad de vida desde la perspectiva de optimismo ante las adversidades, se estableció que el $46,67 \%$ se ubicó en la puntuación de cinco, en la puntuación de totalmente hubo un $6,67 \%$ de personas, mientras que en la respuesta de para nada se presentó un $4 \%$. El promedio de esta pregunta fue de 4,57, el cual es superior a la mediana, demostrando que existe un mayor optimismo ante las adversidades por parte de los colaboradores.

Se evidenció que el $60 \%$ se ubicó en la posición cinco, el 6,67\% consideró que no percibe la felicidad que debería, mientras que el 2,67\% contestó para nada. El promedio fue 4,71 siendo superior a la mediana, esto refleja que las personas si 
perciben la felicidad que deberían tener.

El promedio general de la calidad de vida desde la felicidad fue de 4,66, evidenciando que las personas del área de farmacia se sienten felices; aunque este resultado es cercano a la mediana de cuatro, por lo que el nivel de felicidad no está muy asentado en los colaboradores.

\subsection{Calidad de vida desde la satisfacción con la vida}

Se identificó que el 9,33\% estuvo completamente de acuerdo con que en muchos aspectos, su vida se acerca a su ideal, el $41,33 \%$ estuvo bastante de acuerdo, el $28 \%$ un poco de acuerdo, el 5,33\% un poco en desacuerdo, el $13.33 \%$ bastante en desacuerdo, mientras que el 2,67\% completamente en desacuerdo. El promedio reflejado en esta pregunta es de 4,20, siendo superior a la mediana de 3,50 en una escala del uno al siete, esto refleja que la mayor cantidad de personas respondió afirmativamente en esta pregunta.

En lo que respecta a si las condiciones de vida son excelentes, se pudo identificar que el 5,33\% estuvo completamente de acuerdo, el $38,67 \%$ estuvo bastante de acuerdo, el 32\% un poco de acuerdo, el $16 \%$ un poco de acuerdo, mientras que el $8 \%$ bastante en desacuerdo. No hubo personas que se consideren completamente en desacuerdo; el promedio de esta pregunta fue de 4,17, siendo mayor a la mediana de 3,5 , por lo que se considera que la mayoría respondió de manera afirmativa. La figura 2 muestra el gráfico de barras con las respuestas de esta pregunta. 


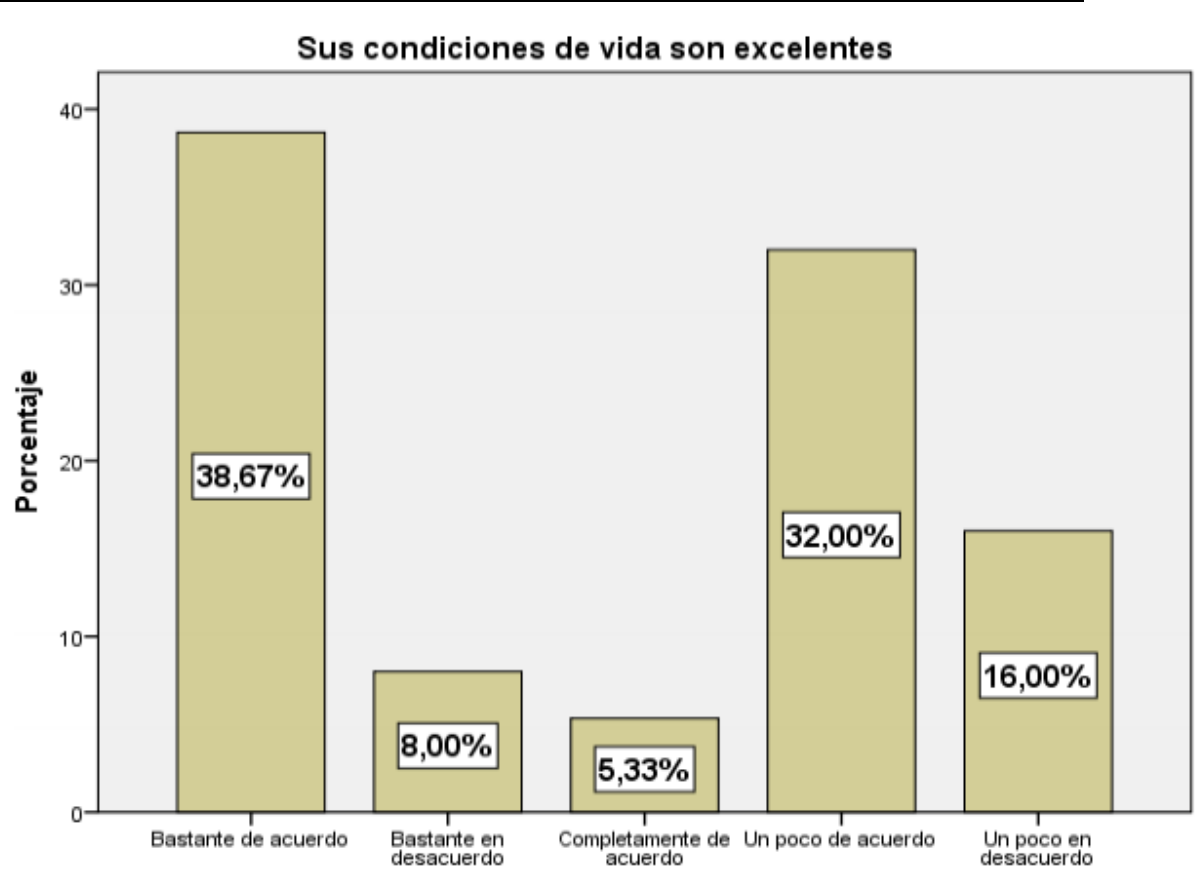

Figura 2. Sus condiciones de vida son excelentes

En la pregunta que se realizó sobre la satisfacción con la vida, las personas respondieron que están completamente de acuerdo en un $21,33 \%$, el $32 \%$ respondió que está bastante de acuerdo, el 37,33\% contestó estar un poco de acuerdo, el $2,67 \%$ un poco en desacuerdo, el $5,33 \%$ bastante en desacuerdo, mientras que el $1,33 \%$ estuvo completamente en desacuerdo. El promedio de esta pregunta fue de 4,57 , siendo superior a la mediana de 3,5, por lo que se considera que la mayoría de las personas respondieron de forma afirmativa a esta pregunta.
En los resultados de la pregunta sobre si ha obtenido cosas importantes que haya deseado en su vida, se identificó que el 10,67\% estuvo completamente de acuerdo, el $42,67 \%$ señaló estar bastante de acuerdo, el $22,67 \%$ un poco de acuerdo, el $9,33 \%$ un poco en desacuerdo, el 10,67\% bastante en desacuerdo, mientras que el $4 \%$ bastante en desacuerdo. El promedio de esta pregunta fue 4,21 , siendo mayor a la mediana de 3,5, por lo que se concluye que la mayoría de las personas respondió afirmativamente ante esta pregunta.

En la pregunta que indica que si tuviera que vivir de nuevo, cambiaría 
algo, el 9,33\% se mostró completamente de acuerdo, el $34,67 \%$ bastante de acuerdo, el $34,67 \%$ un poco de acuerdo, el $8 \%$ un poco en desacuerdo, el $12 \%$ bastante en desacuerdo, mientras que el 1,33\% completamente en desacuerdo. El promedio de esta pregunta fue de 4,17 , superior a la mediana de 3,5 , por lo que se considera que las personas en su mayoría respondieron afirmativamente en esta pregunta.

El promedio general de la dimensión de calidad de vida según la satisfacción de vida reflejó un valor de 4,27, siendo superior a la mediana de 3,5. Este resultado demuestra que la mayoría de colaboradores del área de farmacia se encuentran satisfechos con su vida.

\subsection{Calidad de vida según la satisfacción económica}

En la sección cuatro correspondiente a la calidad de vida según la satisfacción de su situación económica se identificó que el $10,67 \%$ se encuentra completamente satisfecho, el $26,67 \%$ bastante satisfecho, el $10,67 \%$ un poco satisfecho, el 9,33\% un poco insatisfecho, el 39,67\% bastante insatisfecho, mientras que el $4 \%$ completamente insatisfecho.

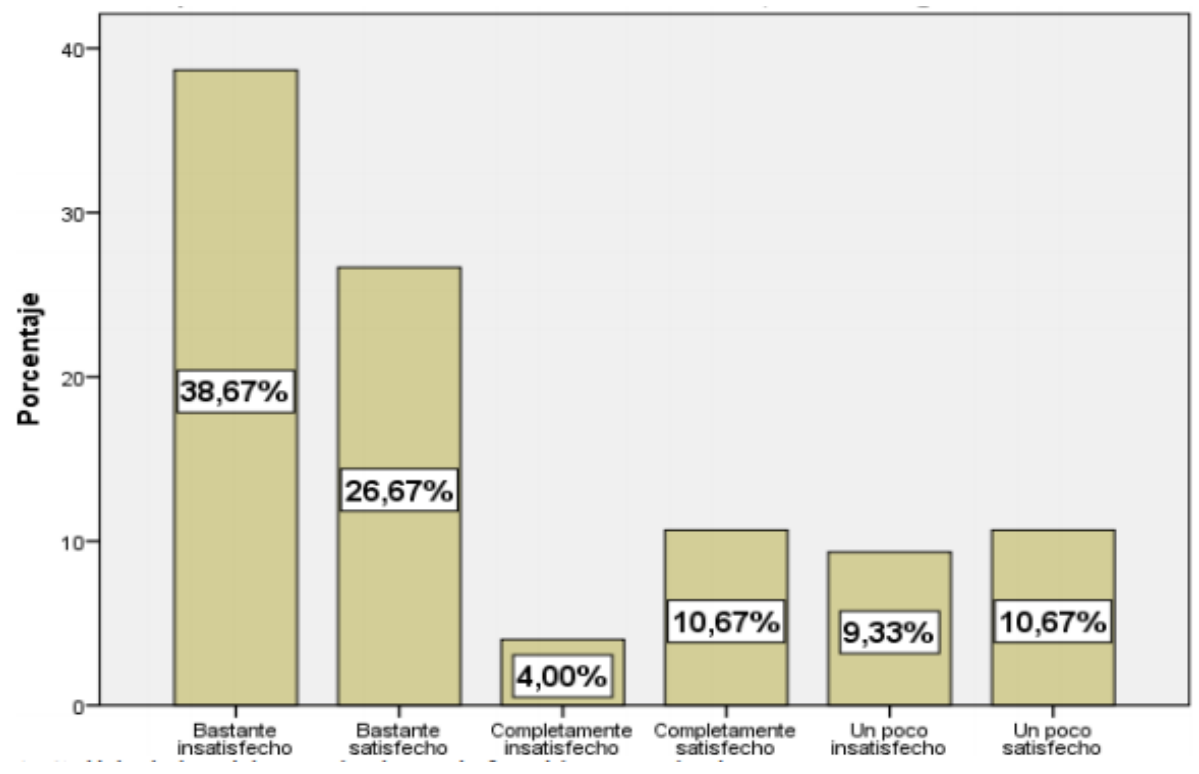

Figura 3. Respecto de su actual situación económica, usted en general está

La figura 3 evidencia que los colaboradores no se encuentran muy a gusto con sus sueldos, el promedio en la escala de Likert de seis niveles 
fue de 3,49, siendo similar a la mediana de 3,5; por tanto, los colaboradores se encuentran divididos en este aspecto.

\subsection{Aspectos sociodemográficos}

Los resultados de la encuesta reflejaron que dentro del área de farmacia del Hospital Teodoro Maldonado Carbo, el 53,33\% son solteros, separados, divorciados 0 viudos; mientras que el $46,67 \%$ son casados o viven en pareja.

En cuanto a los resultados de la encuesta se refleja que el $60 \%$ pertenece al género femenino, en tanto que el $40 \%$ es del género masculino.

\subsection{Calidad de vida según la satisfacción con la vida familiar}

La pregunta sobre si cree que la vida sea absolutamente satisfactoria sin su familia, tuvo una puntuación del $17,33 \%$ para completamente de acuerdo, del $49,33 \%$ para bastante de acuerdo, del $16 \%$ para un poco de acuerdo, del 5,33\% para un poco en desacuerdo, del 10,67\% para bastante en desacuerdo y del 1,33\% para completamente en desacuerdo. El promedio de esta pregunta en base a la escala de Likert de seis niveles fue de 4,53 , lo que es mayor a la mediana de 3,5 , demostrando así que los colaboradores no se imaginan una vida absolutamente satisfactoria sin su familia.

En la pregunta concerniente a si la persona puede ser feliz viviendo sola, se demostró que el $17,33 \%$ se encuentra completamente de acuerdo con esto, el 49,33\% está bastante de acuerdo, el $16 \%$ se ubica como un poco de acuerdo, el $5,33 \%$ un poco en desacuerdo, el $6,67 \%$ bastante en desacuerdo, mientras que el $5,33 \%$ completamente en desacuerdo. El promedio de esta pregunta fue de 4,49 , siendo mayor a la mediana de 3,5 , por lo que los colaboradores pueden ser felices viviendo solos.

En cuanto a la pregunta referente a que si las recompensas de tener a su familia son más importantes que otras cosas, se obtuvo que el $14,67 \%$ está completamente de acuerdo, el 52\% respondió estar bastante de acuerdo, el $16 \%$ estuvo un poco de acuerdo, el $8 \%$ se encontró un poco en desacuerdo, el $8 \%$ se ubicó bastante en desacuerdo, mientras que el 1,33\% estuvo completamente en desacuerdo. El promedio de esta 
pregunta fue de 4,53, siendo mayor a la mediana de 3,5 ; por lo que se considera que los colaboradores respondieron afirmativamente con respecto a esta pregunta.

En la pregunta que tiene por objeto determinar si las necesidades de la familia son más importantes que las propias necesidades del individuo, se determinó que el $12 \%$ estuvo completamente de acuerdo, el $52 \%$ se ubicó como bastante de acuerdo, el $18,67 \%$ un poco de acuerdo, el $8 \%$ un poco en desacuerdo, el $4 \%$ bastante en desacuerdo, mientras que el 5,33\% estuvo completamente en desacuerdo. El promedio total fue de 4,44 , siendo mayor a la mediana de 3,5 , se considera que los colaboradores sienten que las necesidades de su familia son más importantes que las de ellos, tal como se refleja en la figura 4.

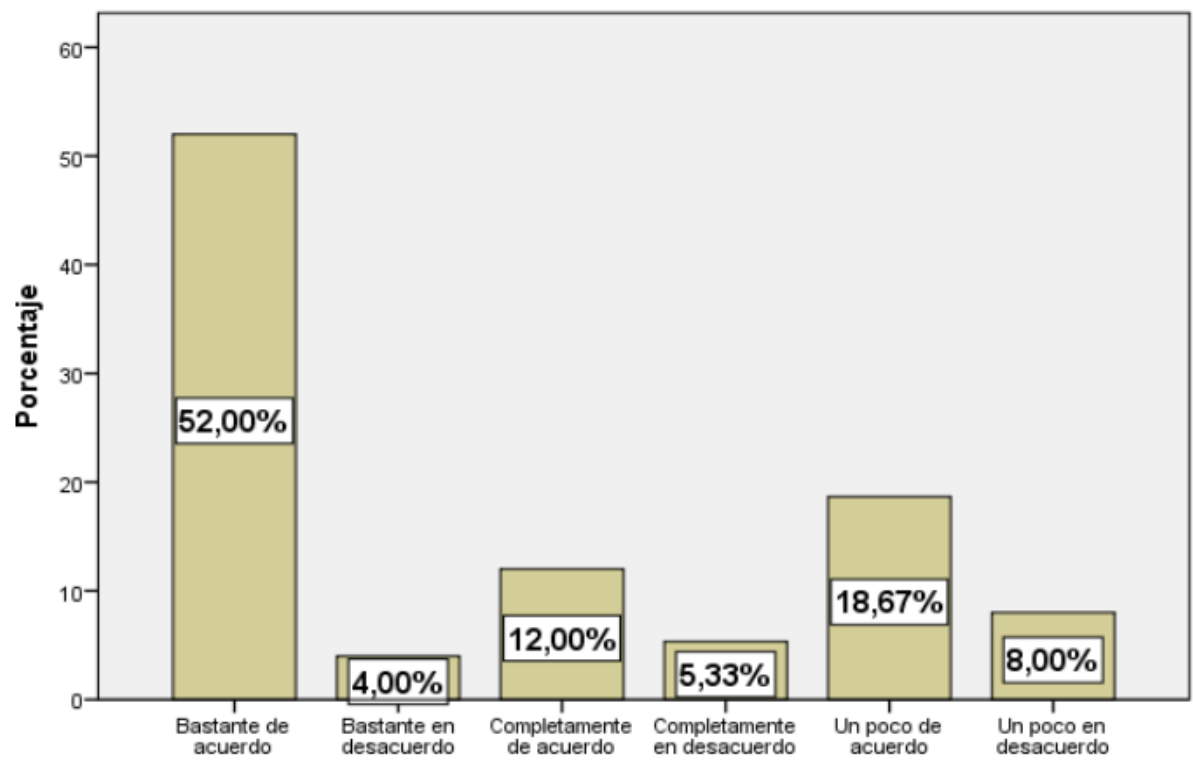

Figura 4. Las necesidades de la familia son más importantes que mis propias necesidades

En la pregunta referente a que las relaciones realmente importantes están en la familia, se evidencia que el $12 \%$ de los empleados estuvo completamente de acuerdo, el $44 \%$ se encontró bastante de acuerdo, el $25,33 \%$ un poco de acuerdo, el $8 \%$ un poco en desacuerdo, el $8 \%$ bastante en desacuerdo, mientras que el 2,67\% contestó estar completamente en desacuerdo. El promedio de esta pregunta fue de 4,36, siendo mayor a la mediana de 3,5; por lo que se puede afirmar que entre de los colaboradores, las 
relaciones realmente importantes están en sus familias.

Por su parte, en la interrogante planteada con respecto a que si las comidas en familia son las actividades más importantes del día, se pudo evidenciar que el $14,67 \%$ estuvo completamente de acuerdo, el $40 \%$ se encontró bastante de acuerdo, el $26,67 \%$ contestó estar un poco de acuerdo, el 9,33\% dijo estar un poco en desacuerdo, el $8 \%$ bastante en desacuerdo, mientras que el $1,33 \%$ se encontró completamente en desacuerdo. El promedio de esta pregunta fue de 4,40 , siendo este valor mayor a la mediana de 3,5; por lo que se demuestra que para los colaboradores las comidas en familia son las actividades más importantes del día. El promedio general de esta dimensión que mide la calidad de vida desde el punto de vista de la satisfacción con la vida familiar fue de 4,46; siendo superior a la mediana de 3,5 , con lo que se demuestra que los colaboradores del área de farmacia se encuentran satisfechos con este aspecto.

3.7 Calidad de vida según el bienestar económico subjetivo

En cuanto a la pregunta sobre el bienestar económico subjetivo, los resultados reflejan que el $49,33 \%$ de los participantes llegaría fácilmente al próximo pago de su sueldo, el $30,67 \%$ consideró que llegaría con algo de dificultad, mientras que el $20 \%$ llegaría con dificultad. EI promedio de esta dimensión en base a la escala de Likert del uno al tres, fue de 2,29, siendo superior a la media de 2, por lo que se considera que no existen demasiadas dificultades para llegar al pago del próximo sueldo (figura 5).

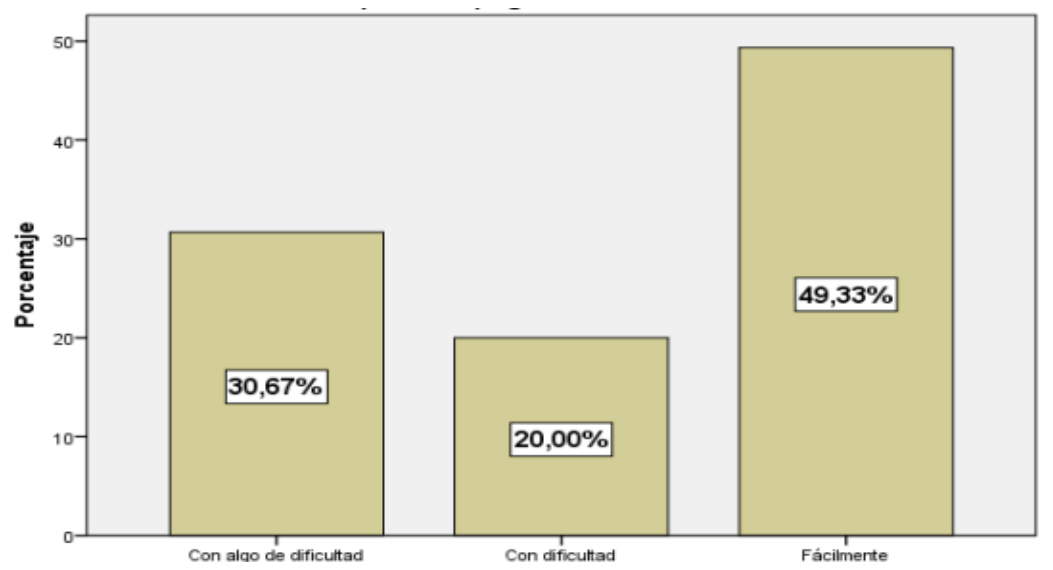

Figura 5. Nivel de dificultad económica para llegar al pago del sueldo 


\subsection{Calidad de vida según la} satisfacción con la vida familiar 2

En esta pregunta se identificó que el $9,33 \%$ estuvo totalmente de acuerdo, el 32\% muy de acuerdo, el $30,67 \%$ de acuerdo, el 9,33\% ni de acuerdo ni en desacuerdo, el 10,67\% en desacuerdo, el $2,67 \%$ muy en desacuerdo, mientras que el 5,33\% totalmente en desacuerdo. El promedio total fue de 4,91, siendo superior a la mediana de la escala de Likert de siete niveles, que corresponde a 4; por tanto, se considera que los colaboradores creen que su vida familiar se acerca a su ideal.

En la pregunta referente a si las condiciones de su vida familiar son excelentes, se pudo identificar que el $9,33 \%$ estuvo totalmente de acuerdo, el $24 \%$ se encontró muy de acuerdo, el $29,33 \%$ estuvo de acuerdo, el $10,67 \%$ en desacuerdo, el $4 \%$ muy en desacuerdo, mientras que el $4 \%$ estuvo totalmente en desacuerdo. El promedio en esta pregunta fue 4,75, siendo mayor a la mediana (4), por lo que se considera que las condiciones de la vida familiar de los colaboradores son propicias.
En la pregunta correspondiente a la satisfacción con la vida familiar, se identificó que el $12 \%$ estuvo totalmente de acuerdo, el $29,33 \%$ muy de acuerdo, el $30,67 \%$ de acuerdo, el $10,67 \%$ en desacuerdo, el $4 \%$ muy en desacuerdo, mientras que el $5,33 \%$ se encontró totalmente en desacuerdo. El promedio en esta pregunta fue 4,91, siendo mayor a la mediana (4); por lo que se considera que los colaboradores respondieron afirmativamente sobre la satisfacción con la vida familiar.

En la pregunta que corresponde a si la persona ha conseguido cosas importantes en su vida familiar, se pudo identificar que el $17,33 \%$ estuvo totalmente de acuerdo, el $22,67 \%$ contestó estar muy de acuerdo, el $28 \%$ se encontró de acuerdo, el $12 \%$ estuvo ni de acuerdo ni en desacuerdo, el $8 \%$ reflejó estar en desacuerdo, el 6,67\% muy en desacuerdo, mientras que el $5,33 \%$ estuvo totalmente en desacuerdo. El promedio de esta pregunta fue 4,88 , siendo mayor a la mediana de 4; por lo que se considera que los colaboradores han contestado afirmativamente con respecto a la consecución de cosas 
importantes que han deseado en su vida familiar.

En la pregunta referente a si la persona puede vivir de nuevo su vida familiar sin hacer cambios, se pudo identificar que el 5,33\% estuvo totalmente de acuerdo, el 22,67\% muy de acuerdo, el $36 \%$ de acuerdo, el $8 \%$ en desacuerdo, el $1,33 \%$ muy en desacuerdo, mientras que el $5,33 \%$ estuvo totalmente en desacuerdo. El promedio de esta pregunta fue 4,71 , siendo superior a la mediana (4), por lo que se considera que los colaboradores respondieron afirmativamente en esta pregunta.

El promedio general de esta dimensión fue 4,83, siendo superior a la mediana (4), por lo que se considera que, en esta segunda escala de la calidad de vida según la satisfacción con la vida familiar, se obtuvieron respuestas positivas de parte de los colaboradores, lo cual se asemeja a los resultados de la primera escala de satisfacción de la vida familiar.

\subsection{Satisfacción laboral escala 1}

A continuación, se presentan los resultados de la primera escala de satisfacción en función del puesto de trabajo; a través del análisis de estos resultados se denota la satisfacción de los funcionarios del Hospital Teodoro Maldonado Carbo, también se mostrarán las causas que provocan insatisfacción en los colaboradores sanitarios.

En la primera escala de satisfacción laboral se obtuvo un promedio de 4,12 en una escala de Likert de seis valores, cuya mediana fue 3,5 ; es decir, la satisfacción está por encima del valor medio, lo cual denota que existe una satisfacción parcial del personal del área de farmacia del Hospital Teodoro Maldonado Carbo. Las principales causas de insatisfacción identificadas fueron la ausencia de reconocimiento por el trabajo bien hecho, la imposibilidad de utilizar las capacidades y la relación entre los directivos y los empleados del área. Las demás opciones fueron la relación con el superior inmediato, con los compañeros, la posibilidad de ascenso y las condiciones físicas del trabajo; por lo que estos son aspectos en los que se debe trabajar para mejorar la satisfacción laboral y por ende la calidad de vida de los colaboradores (ver tabla 2). 


\subsection{Satisfacción laboral escala 2}

El promedio general de la satisfacción laboral según la segunda escala fue 4,33, el cual es superior a la mediana (4) que pertenece a la escala de Likert de los seis niveles de respuesta. Este resultado refleja una satisfacción laboral parcial con respecto al área de enfermería. Entre las mayores causas de insatisfacción identificadas estuvieron el hecho de que el supervisor muestra muy poco interés en los sentimientos de sus subordinados, tener que hacer demasiado en el trabajo y el hecho de sentir que su trabajo no es apreciado. Las otras causas de insatisfacción más importantes fueron el sentir que el trabajo no tiene sentido, el percibir que el supervisor no es lo suficientemente competente en la ejecución de su trabajo, la cantidad de reglas y procedimientos que dificultan el hacer un buen trabajo y el no disfrutar de la relación con los compañeros de trabajo.

\section{Tabla 2}

Preguntas de la primera escala de satisfacción laboral

\begin{tabular}{lc}
\hline Descripción & Media \\
\hline Condiciones físicas del trabajo & 4.11 \\
Libertad para elegir tu propio método de & 4.15 \\
trabajo & \\
Tus compañeros de trabajo & 4.03 \\
Reconocimiento que obtienes por el trabajo & 3.89 \\
bien hecho & \\
Tu superior inmediato & 4.04 \\
Responsabilidad que se te ha asignado & 4.12 \\
Tu salario & 4.12 \\
La posibilidad de utilizar tus capacidades & 3.91 \\
Relación entre dirección y trabajadores en tu & 3.95 \\
empresa & \\
Tus posibilidades de promoción o ascenso & 4.07 \\
El modo en que tu empresa está gestionada & 4.28 \\
La atención que se presta a las sugerencias que & 4.16 \\
haces & \\
Tu horario de trabajo & 4.19 \\
La variedad de tareas que realizas en tu trabajo & 4.41 \\
Tu estabilidad en el empleo & 4.35 \\
Promedio satisfacción laboral 1 & 4.12 \\
\hline
\end{tabular}

Según Chiang \& Krausse (2009) mencionan que en la empresa privada existe una correlación negativa débil, estadísticamente significativa de un $99 \%$ entre antigüedad y las variables satisfacción por el trabajo en general, satisfacción con el ambiente 
físico del trabajo, satisfacción con la forma en que realiza su trabajo, satisfacción con las oportunidades de desarrollo, satisfacción con la relación subordinado-supervisor, satisfacción con la remuneración y la satisfacción con el reconocimiento que recibe de las autoridades. Por su parte, en la empresa pública no se presenta ninguna correlación estadísticamente significativa entre las variables de datos descriptivos de edad, antigüedad y grado con las variables de satisfacción laboral.

En lo que respecta a las relaciones entre los miembros de los grupos de trabajo constituyen un importante factor para la salud personal y organizacional. Se considera la posibilidad de interrelación con los compañeros del trabajo, tal como lo menciona Granados (2011).

Además, Muñoz-Seco et al. (2006) refieren que el clima influye en la calidad de vida profesional, fundamentalmente en la motivación y el apoyo directivo. El compromiso fomenta la motivación intrínseca y la percepción de demandas. La valoración del apoyo directivo mejora si lo hacen la cohesión y el trabajo en equipo.

\section{Conclusiones}

Dentro de las encuestas realizadas con las diferentes escalas para medir la calidad de vida en base a diferentes perspectivas, se pudo evidenciar con mayor rigurosidad su relación con la satisfacción laboral. Se identificó que la calidad de vida desde el punto de vista de la salud tuvo una puntuación mayor a la mediana, lo que indica que la percepción de este aspecto es positiva dentro del personal; en tanto que se mostraron correlaciones positivas media y débil, determinando que existe una relación moderada para esta dimensión.

\section{Bibliografía}

Ávila, J. (2013). ¿Qué es la calidad de vida? Obtenido de http://www.innsz.mx/opencms /contenido/investigacion/comi teEtica/calidadVida.html

Chiang, M. \& Krausse, K. (2009). Estudio empírico de calidad de vida laboral, cuatro indicadores: satisfacción laboral, condiciones y medioambiente del trabajo, organización e indicador global, sectores privado y público. Desarrollo, aplicación y validación del instrumento. Horizontes empresariales, 8(1), 23-50. 
Diener, E., Emmons, A., Larsen, R., \& Griffin, S. (1985). The satisfaction with life scale. Journal of Personality Assessment, 49(1), 71-75. Obtenido de http://labs.psychology.illinois. edu/ ediener/Documents/Die ner-Emmons-LarsenGriffin_1985.pdf

Giarrizzo, V. (2010). Bienestar económico subjetivo: Más allá del crecimiento. Red de Revistas Científicas de América Latina y el Caribe, España y Portugal, 34(28), 934.

doi:http://www.redalyc.org/ht ml/1956/195617795002/

Granados, I. (2011). Calidad de vida laboral: historia, dimensiones y beneficios. Revista de investigación en Psicología, 14(2), 209-221.

Hennessy, C., Moriarty, D., Zack, M., Scherr, P., \& Brackbill, R. (1994). Measuring healthrelated quality of life for public health surveillance. Public Health Rep, 109(5), 665-672. Obtenido de https://www.ncbi.nlm.nih.gov/ pubmed/7938388

Instituto de Hematología e Inmunología. (2018). Metodología de la investigación. Obtenido de http://instituciones.sld.cu/ihi/m etodologia-de-lainvestigacion/

Lozada, R. (2015). La satisfacción laboral no se consigue sólo con un buen salario. Obtenido de

http://www.dgcs.unam.mx/bol etin/bdboletin/2015_403.html

Lyubomirsky, S., King, L., \& Diener, E. (2005). The benefits of frequent positive affect: does happiness lead to success? Psychological Bulletin, 131(6), 803-855.

Muñoz-Seco, E., Coll-Benejam, J. M., Torrent-Quetglas, M., \& Linares-Pou, L. (2006). Influencia del clima laboral en la satisfacción de los profesionales sanitarios. Atención primaria, 37(4), 209214.

https://doi.org/10.1157/13085 951

Pérez, J., \& Fidalgo, M. (1999). NTP 394: Satisfacción laboral: escala general de satisfacción. Obtenido de http://www.insht.es/InshtWeb/ Contenidos/Documentacion/F ichasTecnicas/NTP/Ficheros/ 301a400/ntp_394.pdf

Spector, P. (1997). Job satisfaction: Application, assessment, causes, and consequences. Oakland: Sage Publications, INC.

Universidad de las Américas Puebla. (2018). Metodología. Obtenido de http://catarina.udlap.mx/u_dl_ a/tales/documentos/lad/blanc o_s_nl/capitulo3.pdf

Universitat de Valéncia. (2018). Escala de satisfacción con la 
vida (SWLS). Obtenido de https://www.uv.es/uipd/cuesti onarios/accesolibre/ESV.pdf

Vega, G., Ávila, J., Malagón, A., Calderón, N., Becerril, A., \& Amador, G. (2014). Paradigmas en la investigación. Enfoque cuantitativo y cualitativo. European Scientific Journal, 10(15), 1-6. Obtenido de https://eujournal.org/index.ph p/esj/article/download/3477/3 240

Vera, P., \& Celis, K. (2011). Evaluación de la felicidad: Análisis psicométrico de la escala de felicidad subjetiva en población chilena. Terapia Psicológica, 29(1), 127-133. Obtenido de http://www.redalyc.org/pdf/78 5/78518428013.pdf
Warr, P. (1999). Wellbeing: The Foundations of Hedonic Psychology. New York: Russell Sage Foundation.

Zabriskie, R., \& Ward, P. (2013). Satisfaction with family life scale, marriage \& family. 49(5), $\quad$ 446-463. doi:10.1080/01494929.2013. 768321 